\title{
Computer-assisted and patient-specific 3-D planning and evaluation of a single-cut rotational osteotomy for complex long-bone deformities
}

\author{
J. G. G. Dobbe · K. J. du Pré • P. Kloen • \\ L. Blankevoort · G. J. Streekstra
}

Received: 28 March 2011 / Accepted: 8 September 2011/Published online: 24 September 2011

(C) The Author(s) 2011. This article is published with open access at Springerlink.com

\begin{abstract}
Malunion after long bone fracture results in an incorrect position of the distal bone segment. This misalignment may lead to reduced function of the limb, early osteoarthritis and chronic pain. An established treatment option is a corrective osteotomy. For complex malunions, a single-cut rotational osteotomy is sometimes preferred in cases of angular deformity in three dimensions. However, planning and performing this type of osteotomy is relatively complex. This report describes a computer-assisted method for 3-D planning and realizing a single-cut rotational osteotomy with a patient-specific cutting guide for orienting the osteotomy and an angled jig for adjusting the rotation angle. The accuracy and reproducibility of the method is evaluated experimentally using plastic bones. In addition, complex rotational deformities are simulated by a computer to investigate the relation between deformity and correction parameters. The computed relation between deformity and correction parameters enables the surgeon to judge the feasibility of a single-cut rotational osteotomy. This appears possible for deformities combining axial misalignment with sufficient axial rotation. The proposed 3-D method of preoperative planning and transfer with a patient-specific cutting guide and angled jig renders the osteotomy procedure easily applicable, accurate, reproducible, and is a good alternative for complex and expensive navigation systems.
\end{abstract}

\section{J. G. G. Dobbe $(\bowtie)$ · G. J. Streekstra}

Department of Biomedical Engineering and Physics, Academic Medical Center, University of Amsterdam, Amsterdam, The Netherlands

e-mail: j.g.dobbe@amc.uva.nl

K. J. du Pré · P. Kloen · L. Blankevoort Department of Orthopedic Surgery, Academic Medical Center, University of Amsterdam, Amsterdam, The Netherlands
Keywords Corrective osteotomy - Patient specific · Cutting guide $\cdot$ Virtual planning $\cdot$ Computer assistance

\section{Introduction}

Fractures of the upper and lower limb sometimes result in malunion of the bone segments. Imbalanced joint loading may subsequently lead to chronic pain due to ligament strain and early osteoarthritis $[11,20]$. An established treatment option is a corrective osteotomy $[10,16,17]$. For complex malunions, a single-cut rotational osteotomy is sometimes preferred to restore angular deformity in three dimensions. With a single-cut rotational osteotomy, a cut is made in a specific direction, such, that after the osteotomy the two faces can be rotated with respect to one another, in the plane of the osteotomy, to restore both deflection (axial alignment) and torsion (rotational alignment) of the bone.

A big advantage of a single-cut rotational osteotomy is the fact that it does not require the insertion of an avascular bone graft which may (partially) be resorbed during the healing phase and may consequently lead to non-union or deviation from the planned position [2]. The single-cut rotational osteotomy is also easy to apply, once the cutting plane is oriented accurately. Accurate orientation of the cutting plane requires 3-D imaging and mathematical analysis of a single-cut rotational osteotomy as was clearly described by Sangeorzan et al. [18]. However, a conventional osteotomy is most-often planned using two orthogonal radiographs [15]. This 2-D assessment hides rotations about the long axis of the bone and may cause a misinterpretation of the correction parameters. In addition, it is difficult for a surgeon to judge the feasibility of performing a single-cut rotational osteotomy based on deformity measurements using a protractor. Even when 3-D 
orientation parameters are available, it has been recognized as a challenge for the surgeon [10] to apply the angles for adjusting the orientation of the cutting blade accurately. State of the art methods for general osteotomy feature preoperative planning, intraoperative imaging, and navigation for orienting the osteotomy cut and for accurate positioning of the bones with respect to each other [6, 9, 17, 22]. These complex systems are expensive and not widely available. For all above-mentioned reasons, the single-cut rotational osteotomy is often refrained from, despite its advantages.

This article presents a method for computer-assisted virtual planning and evaluation of a single-cut rotational osteotomy in 3-D using a patient-specific cutting guide for orienting the cutting blade and an angled jig to set the required rotation of the distal bone segment. The method of planning and surgical transfer is evaluated by experiment $(N=4)$ to investigate its accuracy and reproducibility. Since it is generally difficult to judge the feasibility of applying a single-cut rotational osteotomy in developing a treatment plan, we also simulate angular and rotational deformities to investigate the relation between deformity parameters, as measured using a protractor, and correction parameters. This provides insight into the theoretical and practical limitations of the single-cut rotational osteotomy.

\section{Methods}

Home-made planning software [6] was extended, as detailed below, to provide the functionality for planning the orientation of a single-cut rotational osteotomy and for designing a cutting guide. The software enables the user to position the oriented cutting plane interactively along the bone and to add the oblique surface to the cutting guide at that position. For 3-D stereolithography printing, bone and cutting guide polygons are stored to disk.

\subsection{Computer-assisted planning}

The procedure that is used for planning the single-cut rotational osteotomy considers a tibia as target bone and is based on a preoperative CT scan of an affected tibia and of the unaffected contralateral tibia. The latter is mirrored and serves as reference image for planning the correct pose of the affected tibia. The method is therefore limited to cases in which the contralateral side is unaffected. The planning technique is detailed by Dobbe et al. [6] for correcting the distal radius. In short, the affected tibia is segmented and a polygonal description of the bone is extracted for visualization. A double-contour polygon is created by sampling the gray-level CT image $1 \mathrm{~mm}$ towards the inside and outside of the polygon, along the surface normal. Distal and proximal segments are clipped from this double- contour polygon, which are used for alignment (by image registration) with the reference image (Fig. 1). This yields two transformation matrices $\mathbf{M}_{\mathbf{p}}$ and $\mathbf{M}_{\mathbf{d}}$ from which a $4 \times 4$ correction matrix $\mathbf{M}_{\text {corr }}=\mathbf{M}_{\mathbf{p}}^{-1} \mathbf{M}_{\mathbf{d}}$ is calculated [6], which brings the distal contour within the affected image to its corrected state in 3-D space. The matrix $\mathbf{M}_{\text {corr }}$ includes translations and the rotation matrix, in terms of the CT coordinate system. The single-cut rotational osteotomy is generally used to reduce angular and rotational bone deformities. The surgeon has to decide whether residual translations can be compensated for, e.g., by hip joint rotations or a shoe insole. Planning software can be used to assist the surgeon in judging these residual translations [6].

The rotation matrix $\mathbf{R}$, that does not include translations, is extracted from $\mathbf{M}_{\text {corr }}$ and aligns the local coordinate system of the distal bone, C2 (Fig. 2a), with an anatomical coordinate system at the proximal bone segment, C1. Sangeorzan et al. [18], showed that $\mathbf{R}$ can be used to orient the cutting plane in the anatomical coordinate system $\mathrm{C} 1$, perpendicular to a rotation axis in 3-D space also referred to as the helical axis or 3-D deformity axis [12] represented by the direction vector k (Fig. 2b). Rotational alignment is achieved by rotating the distal bone segment over an angle $\beta$ about this axis (Fig. 2b). These parameters are calculated as follows:

$\mathbf{k}=\frac{1}{2 \operatorname{Sin} \beta}\left|\begin{array}{l}r_{23}-r_{32} \\ r_{31}-r_{13} \\ r_{12}-r_{21}\end{array}\right|, \beta=\arccos \left[\frac{1}{2}\left(r_{11}+r_{22}+r_{33}-1\right)\right]$

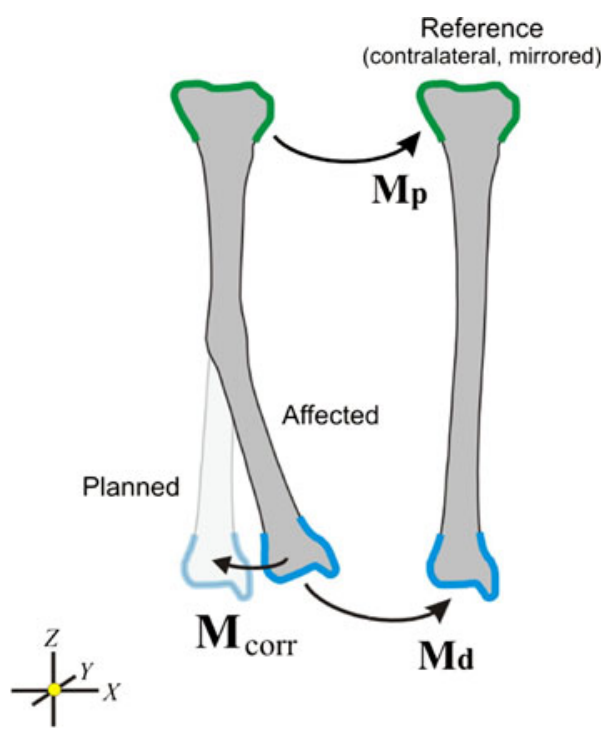

Fig. 1 Affected and reference tibia in a global coordinate system. Distal and proximal double-contour polygons are registered with the reference to provide the correction matrix $\mathbf{M}_{\text {corr. }}$. This is a $4 \times 4$ matrix, which includes translations and rotations, in terms of the CT coordinate system (shown), that brings the distal contour to its corrected state in 3-D space 
(a)

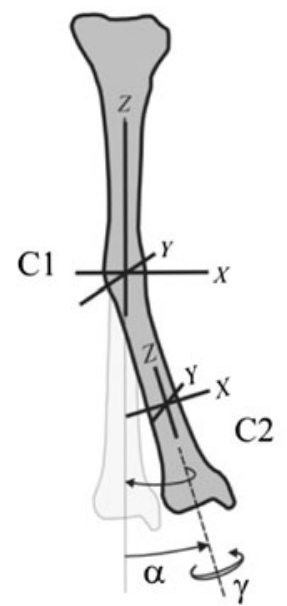

Fig. 2 a Anatomical coordinate system $(\mathrm{C} 1)$ and the mispositioned local coordinate system of the distal end (C2) showing deformity parameters $\alpha$ (deflection) and $\gamma$ (axial rotation). Correcting the distal pose comprises aligning the axes of $\mathrm{C} 1$ with $\mathrm{C} 2$. b The cutting plane is oriented normal to vector $\mathbf{k}$. Rotating the distal segment by $\beta$ about $\mathbf{k}$ compensates bone deflection and torsion. $\mathbf{c}$ The orientation of vector $\mathbf{k}$ can be expressed in terms of the elevation $(\theta)$ and azimuth $(\varphi)$ angles

With : $\mathbf{R}=\left|\begin{array}{lll}r_{11} & r_{12} & r_{13} \\ r_{21} & r_{22} & r_{23} \\ r_{31} & r_{32} & r_{33}\end{array}\right|$

The position of the cutting plane is usually chosen at the fracture site. By rotating the distal segment in the cutting plane over an angle $(\beta)$ about the axis along $\mathbf{k}$, both the deflection and torsion of the bone are corrected. Intraoperative orientation of $\mathbf{k}$ can be facilitated using elevation $(\theta)$ and azimuth $(\varphi)$ angles (Fig. 2c):

$$
\begin{aligned}
& \theta=\arctan \left(\frac{\sqrt{k_{x}^{2}+k_{y}^{2}}}{k_{z}}\right) \\
& \phi=\arctan \left(\frac{k_{y}}{k_{x}}\right)
\end{aligned}
$$

2.2 Surgical method with cutting guide and angled jig

Cutting guides are used in surgical procedures to guide the surgeon in orienting and positioning of the cutting blade [3, $4,7,8,12-14]$. For the rotational osteotomy procedure, a cutting guide is created by cutting a piece of the virtual bone surface and extrusion of that surface into a 3-D mold. The optimal cutting plane, perpendicular to vector $\mathbf{k}$ described above, is manually positioned in virtual space, near the fracture site, and is used to cut the 3-D mold in two pieces. Either of the pieces can be used during surgery as a proximal or distal cutting guide which tightly fits to the bone surface. Such guide features an oblique surface (see virtual representation in Fig. 3b), which is used intraoperatively to guide the surgical saw. The guide is attached to the bone using $\mathrm{K}$ wires. $\mathrm{K}$ wires are also used to help the surgeon in setting the calculated rotation angle $(\beta)$ that achieves rotational alignment. Two $\mathrm{K}$ wires (Fig. 3c, A and B) are drilled into the proximal bone segment and along the cutting plane, to indicate the rotation angle $\beta$. A stainless steel angled jig is prepared preoperatively and is used for setting this angle. A third $\mathrm{K}$ wire $(\mathrm{C})$ is inserted in the distal bone segment, also along the cutting plane, and parallel to $\mathrm{K}$ wire $\mathrm{B}$ (Fig. 3c). Rotational alignment is achieved after osteotomy by rotating the faces of the distal and proximal bone segments until $\mathrm{K}$ wires $\mathrm{C}$ and $\mathrm{A}$ are parallel (Fig. 3d).

\section{Experiments}

\subsection{Assessment of the relation between deformity and correction angles}

It is relatively easy to quantify a deformity in terms of angulation ( $\alpha$, Fig. 2a) and rotation ( $\gamma$, Fig. 2a) parameters during clinical examination. However, it is generally difficult to judge the feasibility of applying a single-cut rotational osteotomy [10] from these deformity parameters. Application of a single-cut osteotomy is practically limited by the steepness of the cut; large osteotomy angles $(\theta$, Fig. 2c) would cleave the tibial ends. In addition, large rotation angles $(\beta$, Fig. 2b) would overstretch surrounding soft tissue, which is also undesirable. Having the relation between deformity parameters $(\alpha, \gamma)$ and correction parameters $(\theta, \beta)$ would help the surgeon in deciding whether a single-cut rotational osteotomy is feasible.

To calculate the relation between deformity and correction parameters, consider a deformed tibia with the proximal bone segment aligned with the $+z$-axis of the $3-\mathrm{D}$ coordinate system $\mathrm{C} 1$, and the distal segment in the $x z$ plane of $\mathrm{C} 1$ (Fig. 2a). Positioning the bone this way eases investigating the correction parameters but does not affect the generality of the solution. The rotation matrix for correction, $\mathbf{R}^{\prime}$ in this case, can be expressed in terms of deformity parameters $(\alpha, \gamma)$ :

$\mathbf{R}^{\prime}=\mathbf{R}_{\mathbf{z}}(\gamma) \mathbf{R}_{\mathbf{y}}(\alpha)$

With $: \begin{aligned} \mathbf{R}_{\mathbf{z}}(\gamma) & =\left[\begin{array}{ccc}\operatorname{Cos} \gamma & -\operatorname{Sin} \gamma & 0 \\ \operatorname{Sin} \gamma & \operatorname{Cos} \gamma & 0 \\ 0 & 0 & 1\end{array}\right] ; \\ \mathbf{R}_{\mathbf{y}}(\alpha) & =\left[\begin{array}{ccc}\operatorname{Cos} \alpha & 0 & \operatorname{Sin} \alpha \\ 0 & 1 & 0 \\ -\operatorname{Sin} \alpha & 0 & \operatorname{Cos} \alpha\end{array}\right]\end{aligned}$

Given this matrix, the correction parameters $\beta=f(\alpha, \gamma)$, $\theta=g(\alpha, \gamma)$ and $\varphi=h(\alpha, \gamma)$, can be calculated using 


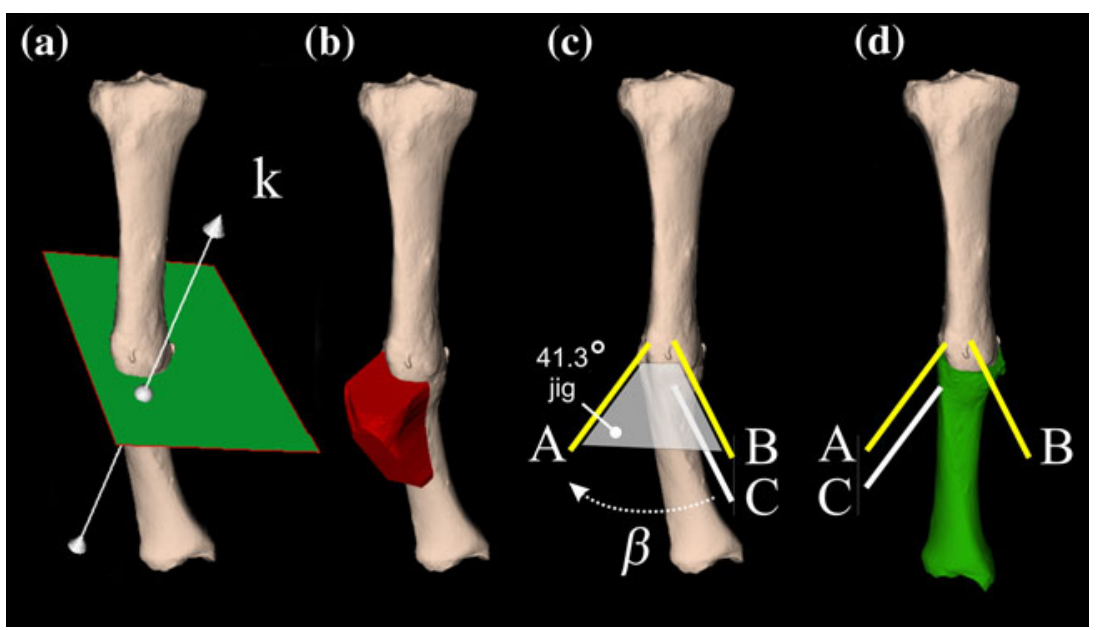

Fig. 3 Osteotomy procedure with cutting guide and jig: a optimal cutting plane is calculated and can be positioned by the user. b Patient-specific cutting guide featuring oblique surface for guiding the cutting blade. c A stainless steel jig serves to set the angle between two Kirschner wires $(A, B)$ in the proximal bone segment. A third wire is inserted into the distal bone segment $(C)$, and parallel to $B$ (the cutting guide is not shown for clarity). d After osteotomy, rotational alignment is achieved by rotating the distal bone segment until $C$ is parallel to $A$. Note that all $\mathrm{K}$ wires are positioned in planes parallel to the optimal cutting plane shown by $\mathbf{a}$
Eqs. $1 \mathrm{~b}, 2 \mathrm{a}$, and $2 \mathrm{~b}$. This enables simulating angular and rotational deformities by varying $\alpha$ over $[0,90]^{\circ}$ and $\gamma$ over $[-180,180]^{\circ}$ and plotting $\beta, \theta$, and $\varphi$.

\subsection{Experimental evaluation of the surgical method}

To investigate the accuracy and reproducibility of the surgical method described above, artificial bones were created of the affected and healthy tibia of a patient suffering from a malunion of the right tibia. To this end, both tibiae were scanned using a Brilliance 64-channel CT scanner (Philips Healthcare, Best, The Netherlands). Voxel spacing was $0.45 \times 0.45 \times 0.45 \mathrm{~mm}$.

Images containing the healthy tibia (mirrored) and the affected tibia were segmented [6] and one plastic model of the healthy reference and four of the affected bone were created using stereolithography printing (SST1200es 3-D printer, Dimension Inc, Eden Prairie, MN), with a resolution of $254 \mu \mathrm{m}$. Plastic bones were printed at a $50 \%$ scale to fit within the physical boundaries of the 3-D printer. A scaled plastic model of the cutting guide was created using the same 3-D printer. To facilitate adequate support of the cutting blade by this scaled cutting guide, the supporting surface was extended to approximately $(\mathrm{L} \times \mathrm{W}): 25 \times$ $18 \mathrm{~mm}$. The outer dimensions of the guide were $(\mathrm{L} \times$ $\mathrm{W} \times \mathrm{H}): 52 \times 18 \times 30 \mathrm{~mm}$. The four plastic models representing the affected tibiae were cut using the guide and the distal, and proximal segments were glued together in the corrected pose after rotational alignment. After completing these experiments, all corrected artificial bones were CT scanned using the same protocol as described above, and the residual errors of the positioning parameters were quantified in 3-D by registration of the distal and proximal segment of each corrected bone with the artificial reference bone that was scanned at the same time.

\section{Results}

\subsection{Assessment of the relation between deformity} and correction angles

For general cases, with the proximal segment aligned with the $+\mathrm{z}$-axis of coordinate system $\mathrm{C} 1$ (Fig. 2a) and the distal segment of the affected bone in the $x z$ plane of coordinate system $\mathrm{C} 1$, correction angles $\beta=f(\alpha, \gamma), \theta=g(\alpha, \gamma)$ and $\varphi=h(\gamma)=0.5 \gamma-90^{\circ}$ were calculated using Eqs. 1b, 2a, and $2 \mathrm{~b}$, for a range of simulated deformities, represented by parameters $(\alpha, \gamma)$. The resulting curves are plotted in Fig. 4a-c. Given the deformation parameters for the specific artificial patient bone, $\alpha \sim 20^{\circ}$ and $\gamma \sim 35^{\circ}$, it can be seen that the correction angles are $\theta \sim 33^{\circ}, \beta \sim 41^{\circ}$, and $\varphi \sim-73^{\circ}$ (represented by the indicated dots; Fig. 4a-c). Figure 4 also shows that the single-cut rotational osteotomy is theoretically possible in all cases, although practical considerations will limit its use (see Sect. 5).

\subsection{Experimental evaluation of the method}

For computer-assisted planning and evaluation, the correction angles are expressed in terms of rotations about the axes of the CT coordinate system (rotation sequence: $y, x, z$ ) 
(a)

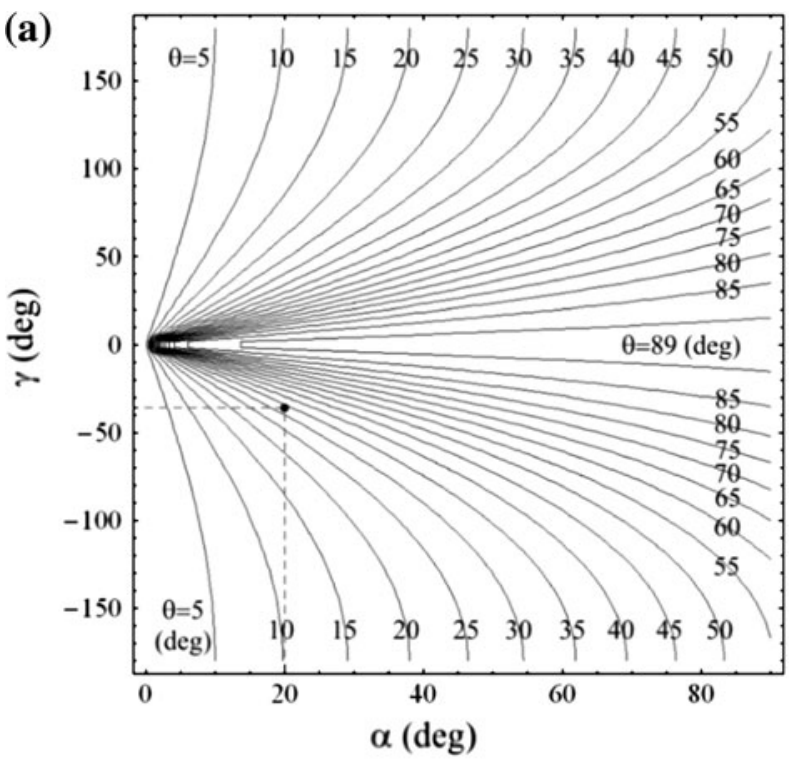

(b)

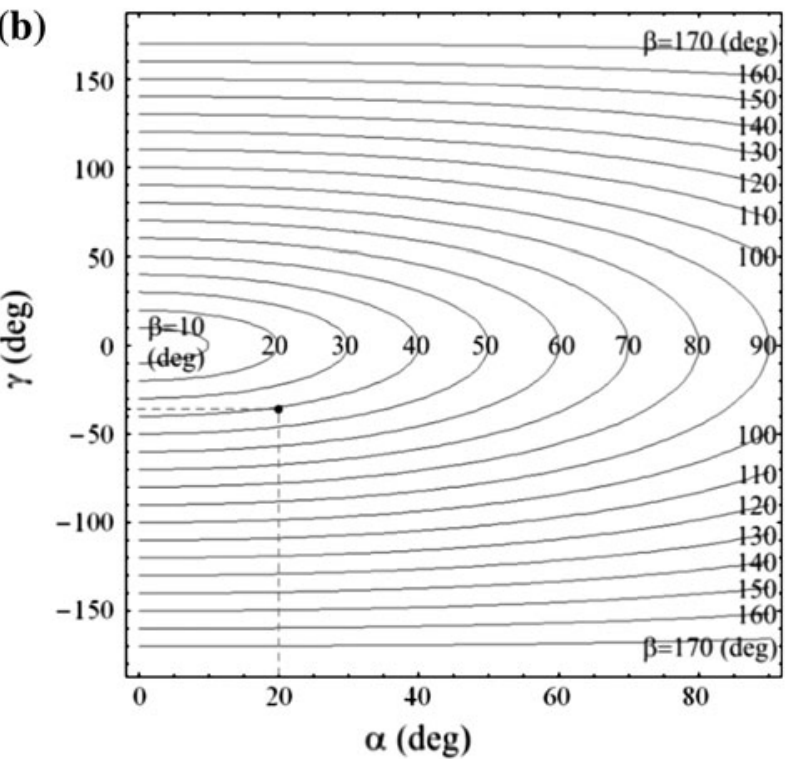

(c)

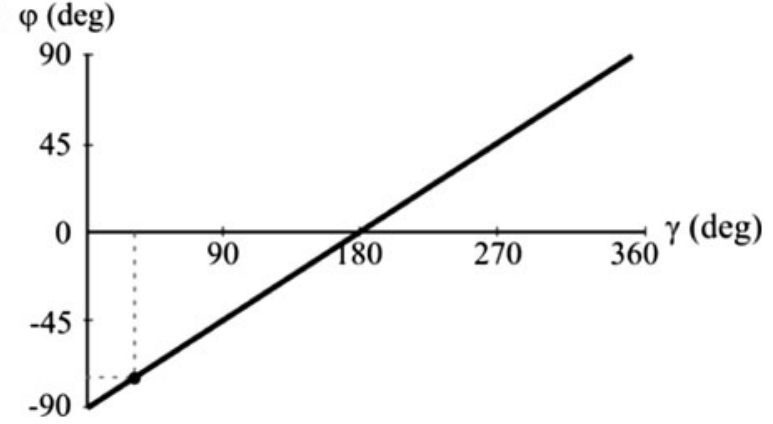

Fig. 4 Relation between correction $(\theta, \beta, \varphi)$ and deformity parameters $(\alpha, \gamma)$ : a Elevation angle $\theta, \mathbf{b}$ rotation $\beta$ and $\mathbf{c}$ azimuth $\varphi$ (see definition in Fig. 2c). The deformation parameters, deflection angle $\alpha$ and torsion $\gamma$, are illustrated in Fig. 2a
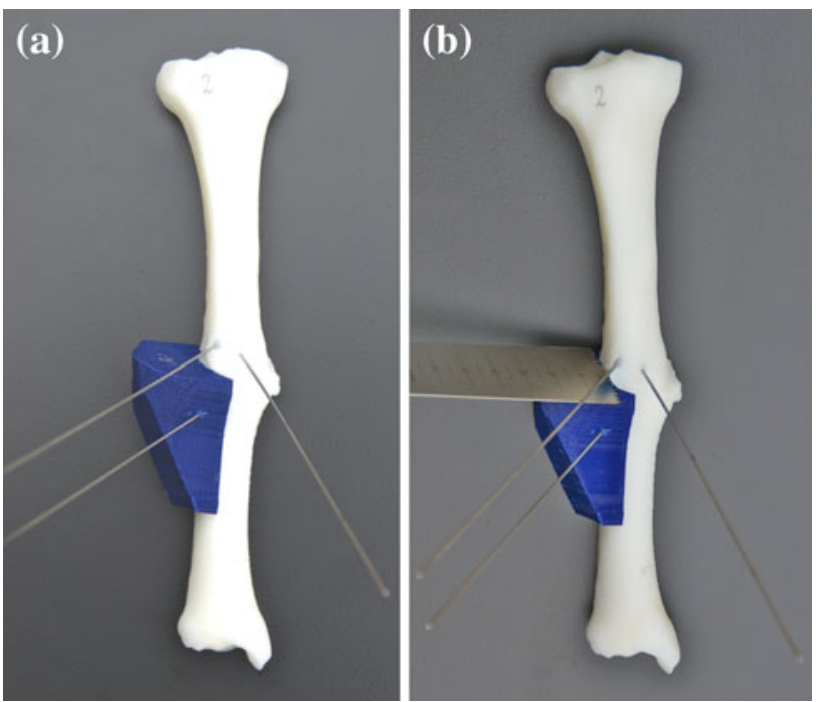

Fig. 5 a Artificial bone specimen for experimental evaluation showing cutting guide (blue) and Kirschner wires after adjusting the rotation angle $\beta$. b Same image, illustrating support of the cutting blade. (Color figure online)

Table 1 Residual rotational errors after osteotomy using a cutting guide and an angular jig

\begin{tabular}{llcl}
\hline Experiment & \multicolumn{3}{c}{ Residual rotational errors (degrees) } \\
\cline { 2 - 4 } & $x$ & $y$ & $z$ \\
\hline 1 & 0.3 & 0.6 & -1.0 \\
2 & 0.1 & 0.9 & -0.6 \\
3 & 0.1 & 1.3 & -1.7 \\
4 & 1.3 & 0.4 & -1.2 \\
Average \pm SD & $0.5 \pm 0.5$ & $0.8 \pm 0.4$ & $1.1 \pm 0.4$ \\
\hline
\end{tabular}

with the $x$-axis pointing in the lateromedial direction, the $y$-axis in the ventrodorsal direction, and the $z$-axis in the proximodistal direction (Fig. 1). The origin of the coordinate system was positioned in the centroid of the distal segment. The required correction angles of the distal segment according to the preoperative planning procedure were $\left(\varphi_{x}, \varphi_{y}, \varphi_{z}\right)=(-19.2,15.6,-32.0)^{\circ}$.

Figure 5 shows an artificial bone specimen that was used for experimental evaluation, with cutting guide attached and $\mathrm{K}$ wires inserted, after adjusting the rotation angle $\beta$ (see also Fig. 3d). The cutting guide snugly fits to the bone, and adjusting the rotation angle $\beta$ using $\mathrm{K}$ wires was very easy. The residual errors of the surgical correction method with a cutting guide and an angled jig are shown in Table 1 . The accuracy of the method is represented by the highest mean deviation $\left(\leq 1.1^{\circ}\right)$ and reproducibility by the highest standard deviation $\left(\leq 0.5^{\circ}\right)$. 


\section{Discussion}

This article described the applicability of a single-cut rotational osteotomy and a method for computer-assisted virtual planning to correct for deflection and torsion in cases of malunited bone deformities. In this method, a computer is used to visualize the affected bone and to automatically calculate the orientation of the osteotomy plane, based on image registration of the proximal and distal bone segment to a reference image containing the unaffected contralateral bone. The surgical correction method, using a cutting guide and angled jig, was evaluated experimentally for intraoperative guidance of the osteotomy direction and for rotating the distal bone segment with respect to the proximal bone segment.

Standard planning of a single-cut rotational osteotomy is based on 2-D radiographs, which is debatable because of its large intra- and inter-observer variation [19] in assessing orientation parameters. In addition, Paley [15] acknowledged the fact that angular deformities, as seen on anteroposterior (AP) and lateral views, are often incorrectly referred to as biplanar angular deformities. He considered the deformities uniangular in an oblique plane [15]. The method proposed by Paley aims on finding the actual bone deflection, which is normally distributed over the AP and lateral projection image. He solved the problem for two degrees of freedom (deflection); however, 2-D images hide a possible rotation about the long axis of the bone. It is therefore impossible to correct for torsional bone deformities using two orthogonal radiographs. The method that we have proposed is based on 3-D CT images which enable finding the arbitrary orientation of the oblique plane of rotational deformity in 3-D. It includes all three degrees of freedom and enables correcting for rotations about the long axis of the bone as well.

It has been shown (Fig. 4) that a single-cut rotational osteotomy is, in theory, always possible. However, very steep osteotomies (large $\theta$ ) are impractical and would cleave the articular surfaces. This occurs in cases of axial misalignment (large $\alpha$ ) with insufficient axial rotation (small $\gamma$; Fig. 4a). In addition, a large correction angle $\beta$ would apply too much strain on the soft tissue. If both a steep osteotomy (large $\theta$ ) and a large rotation angle (large $\beta$ ) are required for appropriate correction, the contact area of the two bone faces would be relatively low. This can be seen from Fig. 6a, which assumes a long bone with a circular cross section. With a steep osteotomy, the faces turn into ellipses with a surface area equal to $\pi r^{2} / \operatorname{Cos}(\theta)$. Rotating these faces about an angle $\beta$ (Fig. 2b) reduces the relative contact area as shown in Fig. $6 \mathrm{~b}$ and requires removing sharp edges of the bone during surgery. For hallux valgus correction, using a linear metatarsal osteotomy, Bettazzoni et al. [1] recommended a bone displacement of less than $60 \%$ of the bone thickness, to guarantee a safe bone union. This translates to an overlapping surface area larger than $\sim 30 \%$, if a circular cross section is considered. Whether or not a deformity can be treated using a rotational osteotomy has to be decided by the surgeon for each individual case. After clinical examination of the deformity using a protractor, Figs. 4 and 6 are helpful in making this decision. Figure 4 is helpful for translating deformity parameters $(\alpha, \gamma)$ into correction parameters $(\beta, \theta)$. Figure 6 helps judging the relative contact area given the correction parameters $(\beta, \theta)$.

Utilization of a patient-specific cutting guide with the use of $\mathrm{K}$ wires to set the rotation angle $\beta$ was easy and accurate. The residual errors are small $\left(\varphi_{\text {err }} \leq 1.1^{\circ}\right)$, and the reproducibility is very high $\left(\mathrm{SD} \leq 0.5^{\circ}\right)$ which is considered acceptable for surgical treatment $[11,20]$. These error values include planning errors and surgical errors. Surgical errors, in orienting the cutting blade and alignment of the bone segments, appear to give the largest contribution to the residual error, since planning proved to be more accurate, with rotation errors as small as $0.12 \pm 0.07^{\circ}$ [6]. The use of similar cutting guides for rotational osteotomy was also described by Murase et al. [12] and Oka et al. [13] of the same group, who used a single guide for inserting parallel pin pairs into both bone segments and for guiding the osteotomy. After the osteotomy, the pin pairs were aligned by application of a second guide, which is an alternative way of applying the rotation angle $\beta$ (Fig. 3c,d). Their method was recently evaluated in 3-D [14] using cadaver specimen showing a similar accuracy and reproducibility for rotational osteotomies $\left(\varphi_{\mathrm{err}} \leq 0.9^{\circ} ; \mathrm{SD} \leq 0.7^{\circ}\right)$. A disadvantage of alignment with a second guide is the fact that it permits a displacement along the pin pairs with respect to each other. In our experience, thin cutting blades sometimes slightly deflect while cutting. If the cut is suboptimal, application of the pin-alignment method fails.

The accuracy and reproducibility of the surgical method with our patient-specific cutting guide and angled jig is superior to other experimental studies that used 3-D navigation-based correction osteotomy methods, such as reported by Croitoru et al. [5] $\left(\varphi_{\mathrm{err}} \leq 4.0^{\circ}\right.$; $\left.\mathrm{SD} \leq 6.5^{\circ}\right)$ and a more recent study by Westphal et al. [22] who showed an overall residual rotation deviation of $4.50^{\circ}$ using an expensive robotic-assisted bone fracture reduction method. A limited number of clinical studies that used computerassisted 3-D planning for correction osteotomy have reported accuracy and reproducibility data. Bilić et al. [2] used 2-D planning to create an intraoperative wedge to be inserted into the osteotomy gap $\left(\varphi_{\mathrm{err}} \leq 3.1^{\circ}\right.$; $\left.\mathrm{SD} \leq 9.6^{\circ}\right)$. Von Campe et al. [21] used true 3-D planning for creation of a similar wedge $\left(\varphi_{\mathrm{err}} \leq 8.0^{\circ} ; \mathrm{SD} \leq 7.5^{\circ}\right)$. The latter two used radiographic (2-D) evaluation of the final orientation 
Fig. 6 a A straight osteotomy of a perfect cylindrical bone results in a circular cross section. An oblique cut yields an elliptical cross section. b Rotating the faces after osteotomy will reduce the relative contact area of these two faces. The required correction angles represent the elevation $(\theta)$ and the rotation $(\beta)$ (see also Fig. 2b, c)
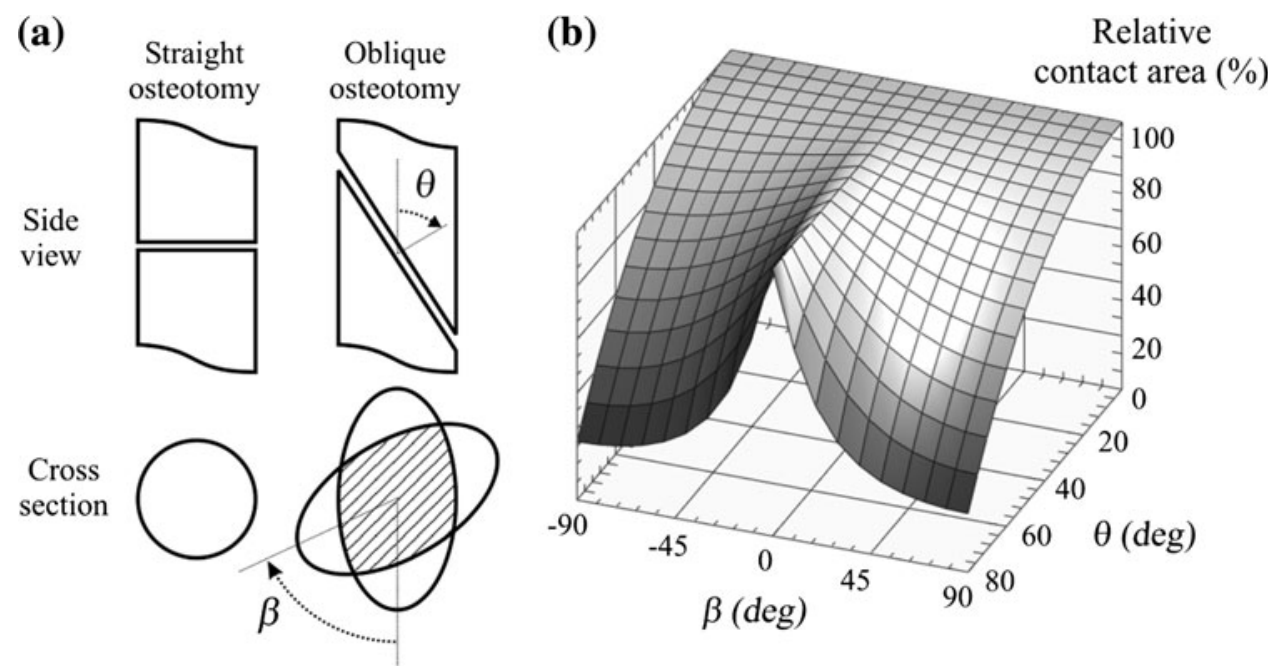

of the distal radius (radial inclination, volar tilt). Although these clinical evaluations showed to be less accurate, further research is required to find the accuracy and reproducibility of our method in a clinical setting.

A limitation of our study is the fact that interobserver variation of the surgical procedure was not investigated. However, interobserver variation can be reduced by giving proper instruction to the surgeons in applying the cutting guide and angled jig. Advantages of the proposed method include the correction in three degrees of freedom, ease of use, and the promising accuracy and reproducibility for surgical treatment.

The computed relation between deformity and correction parameters enables the surgeon to judge the feasibility of applying a single-cut rotational osteotomy. The proposed patient-specific 3-D method of preoperative planning and application of a cutting guide and angled jig renders the osteotomy procedure easily applicable, accurate, and reproducible, and is a good alternative for complex and expensive navigation systems.

Open Access This article is distributed under the terms of the Creative Commons Attribution Noncommercial License which permits any noncommercial use, distribution, and reproduction in any medium, provided the original author(s) and source are credited.

\section{References}

1. Bettazzoni F, Leardini A, Parenti-Castelli V, Giannini S (2004) Mathematical model for pre-operative planning of linear and closing-wedge metatarsal osteotomies for the correction of hallux valgus. Med Biol Eng Comput 42:209-215

2. Bilić R, Kovjanić J, Kolundžić R (2005) Quantification of changes in graft dimension after corrective osteotomy of the distal end of the radius. Acta Chirurgiae Orthopaedicae et Traumatologiae Cechosl. 72:375-380

3. Brown GA, Firoozbakhsh K, DeCoster TA, Reyna JR Jr, Moneim M (2003) Rapid prototyping: the future of trauma surgery? J Bone Joint Surg 85:49-55
4. Ciocca L, De Crescenzio F, Fantini M, Scotti R (2009) CAD/ CAM and rapid prototyped scaffold construction for bone regenerative medicine and surgical transfer of virtual planning: a pilot study. Comp Med Imag Graph 33:58-62

5. Croitoru H, Ellis RE, Prihar R, Small CF, Pichora DR (2001) Fixation-based surgery: a new technique for distal radius osteotomy. Comput Aided Surg 6:160-169

6. Dobbe JGG, Strackee SD, Schreurs AW, Jonges R, Carelsen B, Vroemen JC, Grimbergen CA, Streekstra GJ (2011) Computerassisted planning and navigation for corrective distal radius osteotomy, based on pre- and intraoperative imaging. IEEE Trans Biomed Eng 58(1):182-190

7. Docquier PL, Paul L, Cartiaux O, Delloye C, Banse X (2010) Computer-assisted resection and reconstruction of pelvic tumor sarcoma. Sarcoma 125162:1-8

8. Hafez MA, Chelule KL, Seedhom BB, Sherman KP (2006) Computer-assisted total knee arthrosplasty using patient-specific templating. Clin Orth Rel Res 444:184-192

9. Koo TKK, Chao EYS, Mak AFT (2006) Development and validation of a new approach for computer-aided long bone fracture reduction using unilateral external fixator. J Biomech 39:2104-2112

10. Meyer DC, Siebenrock KA, Schiele B, Gerber C (2005) A new methodology for the planning of single-cut corrective osteotomies of mal-aligned long bones. Clin Biomech 20:223-227

11. Milner SA, Davis TR, Muir KR, Greenwood DC, Doherty M (2002) Long-term outcome after tibial shaft fracture: is malunion important? J Bone Joint Surg Am 84-A(6):971-980

12. Murase $\mathrm{T}$, Kunihiro $\mathrm{O}$, Moritomo $\mathrm{H}$, Goto A, Yoshikawa H, Sugamoto K (2008) Three-dimensional corrective osteotomy of malunited fractures of the upper extremity with use of a computer simulation system. J Bone Joint Surg Am 90(11):2375-2389

13. Oka K, Murase T, Moritomo H, Goto A, Sugamoto K, Yoshikawa H (2010) Corrective osteotomy using customized hydroxyapatite implants prepared by preoperative computer simulation. Int J Med Robotics Comput Assist Surg 6:186-193

14. Oka K, Murase T, Moritomo H, Goto A, Nakao R, Sugamoto K, Yoshikawa H (2011) Accuracy of corrective osteotomy using a custom-designed devise based on a novel computer simulation system. J Orthop Sci 16:85-92

15. Paley D (2005) Principles of deformity correction, 1st edn. Springer, Berlin, Heidelberg, New York. ISBN 3-540-41665-X

16. Patton MW (2004) Distal radius malunion. J Am Soc Surg Hand 4(4):266-274

17. Rieger M, Gabl M, Gruber H, Jaschke WR, Mallouhi A (2005) CT virtual reality in the preoperative workup of malunited distal radius fractures: preliminary results. Eur Radiol 15:792-797 
18. Sangeorzan BP, Judd RP, Sangeorzan BJ (1989) Mathematical analysis of single-cut osteotomy for complex long bone deformity. J Biomech 22(11/12):1271-1278

19. Thomason K, Smith KL (2008) The reliability of measurements taken from computer-stored digitalised X-rays of acute distal radius fractures. J Hand Surg. 33:369-372

20. van der Werken Chr, Marti RK (1983) Post-traumatic rotational deformity of the lower leg. Injury 15:38-40
21. Von Campe A, Nagy L, Arbab D, Dumont CE (2006) Corrective osteotomies in malunions of the distal radius: do we get what we planned? Clin Orthop Relat Res 450:179-185

22. Westphal R, Winkelbach S, Wahl F, Gösling T, Oszwald M, Hüfner T, Krettek C (2009) Robot-assisted long bone fracture reduction. Int J Robotics Res 28(10):1259-1278 